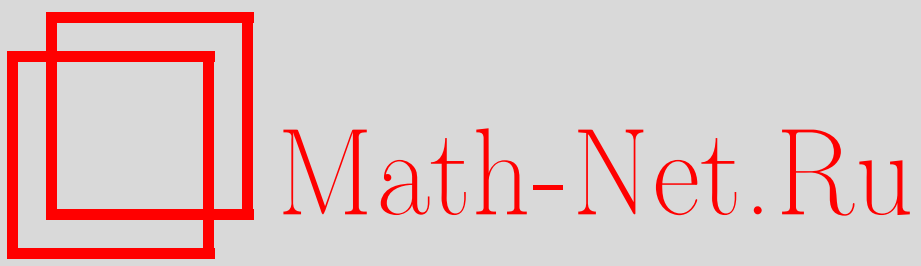

В. А. Васильев, Топологическая сложность и вещественность, Матем. заметки, 1996, том 60, выпуск 5, 670-680

DOI: https://doi.org/10.4213/mzm1880

Использование Общероссийского математического портала MathNet.Ru подразумевает, что вы прочитали и согласны с пользовательским соглашением

http://www. mathnet.ru/rus/agreement

Параметры загрузки:

IP : 54.172 .240 .79

26 апреля 2023 г., 14:04:21

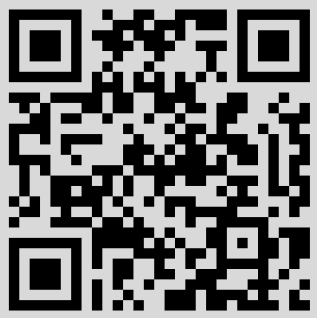




\title{
ТОПОЛОГИЧЕСКАЯ СЛОЖНОСТЬ
}

\section{И ВЕЩЕСТВЕННОСТЬ}

\author{
В. А. Васильев
}

\section{Введение}

Согласно [1], топологическая сложность алгоритма - это число его ветвлений (операторов IF). Топологическая сложность вычислительной задачи - это минимальная топологическая сложность решаюших ее алгоритмов.

В работах [1]-[4] исследовался этот показатель для задачи приближенного решения комплексньх полиномиальных уравнений и систем, в частности, в [2] было доказано, что топологическая сложность задачи приближенного нахождения всех корней комплексного полинома вида

$$
x^{d}+a_{1} x^{d-1}+\cdots+a_{d}
$$

асимптотически равна $d-1$ (и равна в точности $d-1$, если $d-$ степень простого числа). Настоящая работа посвящена вешественному аналогу этой проблемы: мы исследуем топологическую сложность приближенного нахождения одного вещественного корня вешественного полинома вида (1).

Мы используем следующее определение алгоритма (см. [1]). Алгоpuтм - это ориентированньй граф с узлами следуюших четырех типов: единственный вход (в нашем случае входные данные - это $d$ чисел $\left.a_{i}\right)$; вычислительные узлы, в которых вьполняются арифметические операции над исходньми данными и числами, полученными в алгоритме ранее; узль ветвления, в которых одно из чисел, найденных выше, сравнивается с нулем, и управление передается в зависимости от результата сравнения; выходы, в которых какие-то из полученных ранее чисел объявляются решениями задачи. Поскольку алгоритм - дерево, его топологическая сложность на 1 меньше числа выходов. Алгоритм

Работа выполнена при поддержке Российского фонда фундаментальных исследований, грант № 95-01-00846а, и фонда INTAS, грант № 4373. 
полиномиален, если его вычислительные узлы содержат лишш операции сложения, умножения и взятия постоянной функции.

Обозначим через $B^{d}$ множество вещественных полиномов вида $(1)$, все комплексные корни которых лежат в круге $|z| \leqslant 1$. Соответствующая задача $\Pi(\varepsilon, d)$ состоит в том, чтобы любому полиному $f \in B^{d}$ сопоставить вещественное число, находящееся в $\varepsilon$-окрестности некоторого вещественного корня $f$. Топологическая сложность этой задачи обозначается через t.c. $(\varepsilon, d)$, a ее предельное значение $\lim _{\varepsilon \rightarrow+0}$ t.c. $(\varepsilon, d)$ - через t.c. $(d)$.

Конечно, эта задача корректна, лишш если $d$ нечетно; аналогичная задача, имеющая смысл для любых $d$, состоит не в приближенном поиске некоторого корня всякого полинома $f \in B^{d}$, а в поиске его $\varepsilon$-корня, т.е. такой точки $x \in \mathbb{R}^{1}$, что $|f(x)| \leqslant \varepsilon$ (см. [3]). Именно, для любых $\varepsilon>\varepsilon^{\prime}>0$ алгоритм решает задачу $\Pi_{\approx}\left(\varepsilon, \varepsilon^{\prime}, d\right)$, если для любого $f \in B^{d}$ он выдает на выходе либо $\varepsilon$-корень $f$, либо верное утверждение о том, что $f$ не имеет $\varepsilon^{\prime}$-корней.

ПРЕДЛОЖЕНИЕ 1. Функиия t.с.(·) монотонна: если $d^{\prime}>d-\partial в а$ нечетных числа, то для любого $\varepsilon$ t.c. $\left(d^{\prime}, \varepsilon\right) \geqslant$ t.c. $(d, \varepsilon)$.

Действительно, алгоритм, решаюший задачу $\Pi\left(\varepsilon, d^{\prime}\right)$, в частности, находит вещественный корень любого полинома вида $\left(x^{2}+1\right)^{\left(d^{\prime}-d\right) / 2} f$, $f \in B^{d}$.

ТЕорема 1. 1. Для любых $\varepsilon>\varepsilon^{\prime}>0$ любого четного $d=2 m$ топологическая сложность задачи $\Pi_{\approx}\left(\varepsilon, \varepsilon^{\prime}, d\right)$ не превосходит $m$.

2. При достаточно малых $\varepsilon$ эта топологическая сложность не меньие $m$.

ТЕорема 2. Для любого достаточно малого $\varepsilon>0$ t.c. $(\varepsilon, 3) \geqslant 1$.

ТЕОремА 3. Для любого нечетного $d$ и любого $\varepsilon>0$ существует полиномиальный алгоритм топологической сложсности $[d / 2]$, решающий задачу $\Pi(\varepsilon, d)$.

СлЕДСТВИЕ. t.c.(3) $=1$.

Оценка из теоремы 3 нереалистична уже при $d=5$.

ТЕОРемА 4. Для любого $\varepsilon>0$ существует полиномиальный алгоритм топологической сложнооти 1 , решающий задачу $\Pi(\varepsilon, 5)$.

ЗАМЕЧАнИЕ. Все перечисленные результаты о сложности задач $\Pi(\varepsilon, d), \Pi_{\approx}\left(\varepsilon, \varepsilon^{\prime}, d\right)$ останутся справедливы, если мы заменим множество $B^{d}$ любой ограниченной областью в $\mathbb{R}^{d}$, содержащей окрестность полинома $x^{d}$. 


\section{§1. Доказательство теорем 2 и 3}

Рассмотрим пространство $\mathbb{R}^{2}$ полиномов $x^{3}+a x+b$; пусть $\Theta$ - маленькая окружность с центром в точке 0 этого пространства, так что $\Theta \subset B^{3}$. Рассмотрим множество $M_{3} \subset \Theta \times \mathbb{R}^{1}$, состоящее из всех пар вида \{полином $f \in \Theta$; вещественный корень $f\} . M_{3}$ диффеоморфно $S^{1}$; ограничение на $M_{3}$ очевидной проекции $\Theta \times \mathbb{R}^{1} \rightarrow \Theta$ имеет две точки складки над полиномами $f$, имеюшими кратные корни (см. рис. 1 ).

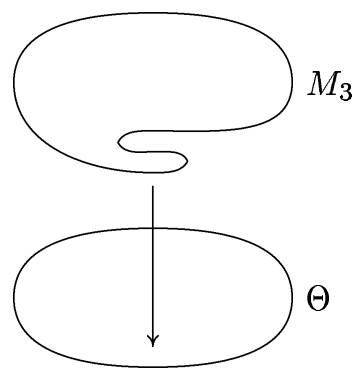

Рис. 1. Проекция $M_{3} \rightarrow \Theta$ не имеет приближенных сечений

Допустим, что t.c. $(\varepsilon, 3)=0$, т.е. существует алгоритм без ветвлений, решаюший нашу задачу. Для любых начальных данных $f=(a, b) \in \Theta$ этот алгоритм дает на выходе некоторое число $\alpha(f)$; функция $\{f \rightarrow \alpha(f)\}$ непрерьвна на $\Theta$. Для любого $f$ значение $\alpha(f)$ должно лежать в $\varepsilon$-окрестности некоторой точки прообраза $f$ при проекции $M_{3} \rightarrow \Theta$. При малых $\varepsilon$ это невозможно и теорема 2 доказана.

ЗАмЕчАнИЕ. Несложный анализ этого доказательства дает точное условие на $\varepsilon$ : утверждение теоремы 2 справедливо для любого положительного $\varepsilon<1 / 2$.

ДоКАЗАТЕЛЬСТВо ТЕОРЕМЫ 3 . Для Любого $i=1,3,5, \ldots, d$ обозначим через $V_{i}$ множество всех полиномов $f \in B^{d}$, имеющих ровно $i$ вещественных корней (с учетом их кратностей), и через $W_{i}$ - множество $V_{i} \cap V_{i+2} \cap \cdots \cap V_{d}$.

На всяком $V_{i}$ определена непрерьвная функция $\{f \rightarrow$ наибольший вещественный корень $f\}$; она продолжается до непрерывной функции на замыкании $V_{i}$, а следовательно, также и на некоторой открытой окрестности $\tilde{V}_{i}$ этого замькания в $W_{i}$. Эта окрестность может быть выбрана настолько близкой к $V_{i}$, что для любой ее точки $f$ значение этой продолженной функции лежит в $\varepsilon / 2$-окрестности некоторого вещественного корня $f$. По теореме Вейерштрасса найдется полином $\varphi_{i}: \mathbb{R}^{d} \rightarrow \mathbb{R}, \varepsilon / 2$-аппроксимируюший эту функцию всюду в $\tilde{V}_{i}$, и при $i<d$ полином $\chi_{i}$ такой, что $V_{i} \subset\left\{\chi_{i}<0\right\}$ и $W_{i+2} \backslash \tilde{V}_{i} \subset\left\{\chi_{i}>0\right\}$. Искомьй алгоритм выглядит так. 
Вводим $f$. Вычисляем $\chi_{1}(f) \cdot \chi_{1}(f)>0$ ? Если нет, вычисляем $\varphi_{1}(f)$ и выдаем результат на выход. Если да, вычисляем $\chi_{2}(f) \cdot \chi_{2}(f)>0$ ? Если нет, вычисляем $\varphi_{2}(f)$ и идем на выход. Если да, вычисляем $\chi_{3}(f)$ и т.д.

\section{§2. Доказательство теоремы 1}

Доказательство части 1 этой теоремы аналогично доказательству теоремы 3 , доказательство части 2 основано на следующем утверждении (cp. [1]).

ПРЕДЛОЖЕНИЕ 2. Пусть существует алгоритм топологической сложности $k$, решающий задачу $\Pi_{\approx}\left(\varepsilon, \varepsilon^{\prime}, d\right)$. Тогда для любых положительных чисел $\tilde{\varepsilon}$ и $\tilde{\varepsilon}^{\prime}$ таких, что $\tilde{\varepsilon}>\varepsilon, \varepsilon^{\prime}>\tilde{\varepsilon}^{\prime}$, найдется покрытие $B^{d} k+1$ открытым множеством $U_{0}, U_{1}, \ldots, U_{k}$ и непрерывные функиии $\varphi_{i}: U_{i} \rightarrow \mathbb{R}, i=1, \ldots, k$, такие, что

а) $U_{0}$ принадлежит множеству полиномов, не имеющих $\tilde{\varepsilon}^{\prime}-\kappa о р-$ ней;

b) для любого $i=1, \ldots, k$ и любого $f \in U_{i}\left|f\left(\varphi_{i}(x)\right)\right| \leqslant \tilde{\varepsilon}$.

Доказательство, по сушеству, повторяет доказательство теоремы А из [1]. В частности, $U_{0}$ - это малая окрестность множества тех начальных данных, подав которые на вход, мы выйдем через выход, на котором написано "нет $\varepsilon^{\prime}$-корней"; для любого из оставшихся выходов (число которых не превосходит $k$ ) соответствуюшее множество $U_{i}$ - это малая окрестность множества тех $f$, подав которые на вход, мы выйдем именно через этот выход.

Пусть $d=2 m$ и $\alpha_{1}<\alpha_{2}<\cdots<\alpha_{m}$ - некоторые $m$ точек интервала $(-1,1)$. Определим $\Omega \subset B^{d}$ как множество полиномов $f$, имеющих $m$ локальных минимумов в точках $\alpha_{i}$ и таких, что $\left|f\left(\alpha_{i}\right)\right| \leqslant 2 \tilde{\varepsilon}$.

Лемма 1. Если г достаточно мало по сравнению с минимальным расстоянием между $m+2$ точками $\alpha_{1}, \ldots, \alpha_{m}, 1,-1$, то

а) для любого $f \in \Omega$ множество $\{x|| f(x) \mid \leqslant 2 \tilde{\varepsilon}\}$ состоит из $m$ непересекающихся отрезков (быть можсет, вырожденных), содер жащих точки $\alpha_{i}$;

b) $m$ функиий на $\Omega$, значения которых в точке $f$ равны $f\left(\alpha_{i}\right) / \tilde{\varepsilon}$, $i=1, \ldots, m$, задают гомеоморфизм многообразия $\Omega$ на куб

$$
\square^{m}=[-2,2]^{m} .
$$

ДокАЗАТЕЛЬСТво элементарно.

Пусть $t_{1}, \ldots, t_{m}$ - стандартные координаты в $\mathbb{R}^{m}$. 
ЛЕмма 2. Допустим, что имеется покрытие $B^{d}$ областями $U_{i} u$ функиии $\varphi_{i}$, удовлетворяющие условиям из предложения 2 с достаточно малыми $\tilde{\varepsilon}>\tilde{\varepsilon}^{\prime}$. Тогда существует покрытие куба (2) $k+1$ открытым множеством, связные компоненты которых помечены числами $0,1, \ldots, m$ таким образом, что

а) для любого $i=1, \ldots, m$ слой $\left\{t \mid t_{i}>1\right\} \subset \square^{m}$ не пересекается с областями, помеченными индексом $i$;

b) все области, помеченные нулем, лежат в кубе $\left\{t \mid t_{i} \in\left[\tilde{\varepsilon}^{\prime} / \tilde{\varepsilon}, 2\right]\right.$ $\forall i\}$ (c.м. puc. 2a).

Действительно, эти открытые множества суть пересечения $\Omega$ с областями $U_{i}$ из предложения 2 ; связная компонента такого множества помечена числом 0 , если это компонента множества $\Omega \cap U_{0}$, и числом $i>0$, если значения функции $\varphi_{i}$ в ее точках принадлежат отрезку, содержашему точкy $\alpha_{i}$.

Вершина $(2, \ldots, 2)$ (соответственно $(-2,2, \ldots, 2)$, соответственно $(2$, $-2,2, \ldots, 2)$ и т.д.) нашего куба может быть покрыта только компонентами, помеченными числом 0 (соответственно 1 , соответственно 2 и т.д.), поэтому мы обозначим эти $m+1$ вершин через [0], [1], [2] и т.д.

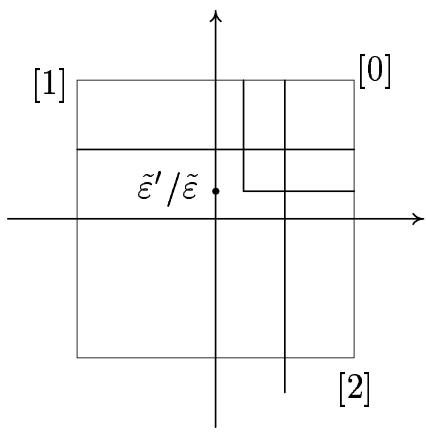

a

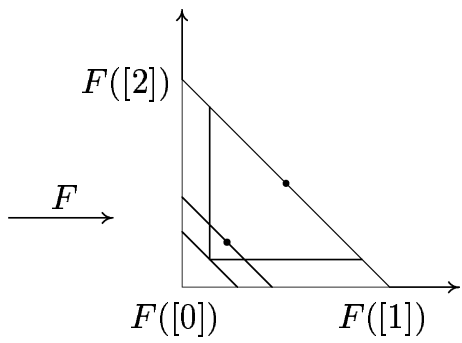

$\mathrm{b}$

Рис. 2. Куб $\square^{2}$ и отображение $F$

ПРЕДЛОЖЕНИЕ 3. Если имеется нумерованное покрытие множества $\Omega$, удовлетворяюшее условиям леммы 2 , то найдется точка куба (2), покрытая областями, помеченными всеми $m+1$ возможнными числами (и, следовательно, принадлежащими различным множествам $\left.U_{i}\right)$. В частности, в әтом случае $k \geqslant m$.

Теорема 1 немедленно вытекает из этого предложения, доказательство которого основано на следующей лемме. 
ЛЕмма 3. Если непрерывное отображсение т-мерного симплекса $\Delta^{m}$ в себя переводит каждую грань в себя, то его образ совпадает $c \Delta^{m}$ (и, более того, степень индуцированного отображения сферь $\Delta^{m} / \partial \Delta^{m}$ в себя равна 1$)$.

Это немедленно доказывается индукцией по размерности граней.

Теперь мы построим два отображения $F, G$ куба $\square^{m}$ на симплекс $\Delta^{m}$, первое из которых является гомеоморфизмом.

1. Сначала выполним аффинное преобразование $\mathbb{R}^{m} \rightarrow \mathbb{R}^{m}$, отображающее вершины $[0],[1], \ldots,[m]$ куба $\square^{m}$ соответственно в центр 0 и концы $(1,0, \ldots, 0), \ldots,(0, \ldots, 0,1)$ базисного репера. Затем отобразим образ куба $\square^{m}$ (т.е. куб $\left\{0 \leqslant x_{i} \leqslant 1\right\}$ ) на симплекс $\Delta^{m}=\left\{x_{1} \geqslant 0, \ldots, x_{m} \geqslant 0\right.$, $\left.\sum x_{i} \leqslant 1\right\}$ кусочно-линейным гомеоморфизмом главного октанта $\mathbb{R}_{+}^{m}$ на себя, сохраняюшим каждую прямую, проходящую через начало координат, и линейным в ограничении на всякую такую прямую (см. рис. $2 \mathrm{~b}$ ). Искомьй гомеоморфизм $F: \square^{m} \rightarrow \Delta^{m}$ является композицией этих двух.

2. Пусть $\left\{\chi_{i}\right\}, i=0,1, \ldots, m,-$ произвольное разбиение единицы, подчиненное нашему покрытию $\square^{m}$, такое, что $\chi_{i} \equiv 0$ вне областей, помеченных числом $i$. Для любого $i=1, \ldots, m$ определим $\lambda_{i}: \square^{m} \rightarrow \mathbb{R}^{m}$ как (разрьвное) отображение, переводящее объединение таких областей в базисный вектор $e_{i}$, а дополнение к таким областям - в начало координат; определим $\lambda_{0}$ как тождественное отображение, переводящее $\square^{m}$ в начало координат пространства $\mathbb{R}^{m}$. Тогда искомое отображение $G: \square^{m} \rightarrow \mathbb{R}^{m}$ определяется как $\sum_{i=0}^{m} \chi_{i} \lambda_{i}$.

По построению нашего нумерованного покрытия $\square^{m}$, отображение $G \circ F^{-1}: \Delta^{m} \rightarrow \Delta^{m}$ удовлетворяет условиям леммы 3 . По определению разбиения единишы, любая точка прообраза внутренности $\Delta^{m}$ при этом отображении принадлежит областям, помеченным всеми $m+1$ индексами, и предложение 3 доказано.

\section{§3. Доказательство теоремы 4}

3.1. Первые упрощения. Для того чтобы найти число t.c. $(d)$, достаточно решить аналогичную проблему лишь для множества полиномов (1) c $a_{1}=0$ : действительно, все полиномы сводятся к таким сдвигом аргумента $x$ (этот сдвиг не использует ветвлений и увеличивает максимальное абсолютное значение комплексных корней $f$ менее чем вдвое).

На оставшемся пространстве $\mathbb{R}^{d-1}$ полиномов вида

$$
x^{d}+a_{2} x^{d-2}+\cdots+a_{d}
$$


действует однопараметрическая группа $G \simeq \mathbb{R}_{+}$растяжений переменной $x$ :

$$
G_{t}(f)=t^{d} f(x / t), \quad G_{t}\left(a_{2}, a_{3}, \ldots, a_{d}\right)=\left(t^{2} a_{2}, t^{3} a_{3}, \ldots, t^{d} a_{d}\right),
$$

$t \in(0, \infty)$. Пусть $\Theta$ - произвольная гиперповерхность в $\mathbb{R}^{d-1}$, диффеоморфная $S^{d-2}$, отделенная от 0 и такая, что любая орбита группы (4) пересекает ее трансверсально в единственной точке.

ПРЕДЛОЖЕНИЕ 4. Пусть для любого $\varepsilon>0$ существует покрытие поверхности $\Theta k$ открытыми подмножествами $U_{1}, \ldots, U_{k}$ и система непрерывных функций $\varphi_{i}: \overline{U_{i}} \rightarrow \mathbb{R}, i=1, \ldots, k$, такая, что для любого $f \in U_{i}$ значение $\varphi_{i}(f)$ лежит в $\varepsilon$-окрестности некоторого корня полинома $f$. Тогда для всякого $\varepsilon^{\prime}>0$ найдется полиномиальный алгоритм топологической сложности $k-1$, решающий задачу $\Pi\left(\varepsilon^{\prime}, d\right) ;$ в частности, t.c. $(d) \leqslant k-1$.

Это следует немедленно из аппроксимационной теоремы Вейерштрасса.

В дальнейшем мы рассматриваем случай $d=5$ и строим такое покрытие $\mathrm{c} k=2$. При этом мы отождествляем $\Theta$ с фактором пространства $\mathbb{R}^{4} \backslash 0$ по действию группы (4) и обозначаем через $\{g\}$ точку поверхности $\Theta$, в которой она протыкается орбитой, содержашей точку $g \in \mathbb{R}^{4}$.

3.2. Симплекс $\Delta$. Обозначим через $\Delta$ пересечение топологической сферы $\Theta \subset \mathbb{R}^{4}$ с замыканием множества полиномов (3), имеющих 5 вещественных корней (с учетом кратностей). Это пересечение гомеоморфно трехмерному симплексу. Именно, если $x_{1} \leqslant \cdots \leqslant x_{5}$ - все корни полинома $f \in \Delta$, то координатами точки $f$ в $\Delta$ являются три числа $\left(x_{2}-x_{1}\right) /\left(x_{5}-x_{1}\right)$, $\left(x_{3}-x_{1}\right) /\left(x_{5}-x_{1}\right)$ и $\left(x_{4}-x_{1}\right) /\left(x_{5}-x_{1}\right)$. Мы обозначаем эти числа через $b(f), c(f)$ и $d(f)$ соответственно; очевидно, они лежат в симплексе $0 \leqslant b \leqslant c \leqslant d \leqslant 1$ в $\mathbb{R}^{3}$ (см. рис. 3 ).

ЗАмечаниЕ. Множество $\Delta$ необъемлемо диффеоморфно симплексу. Действительно, его граница имеет ребра возврата в отрезках $(b=c=0)$, $(b=c=d)$ и $(c=d=1)$ и ласточкины хвосты - в вершинах $(b=c=d=0)$ и ( $b=c=d=1)$. В окрестности этих ребер (соответственно вершин) три (соответственно четыре) корня соответствующих полиномов очень близки друг к другу, именно, это корни $\left(x_{1}, x_{2}, x_{3}\right),\left(x_{2}, x_{3}, x_{4}\right)$ и $\left(x_{3}, x_{4}, x_{5}\right)$ для ребер и $\left(x_{1}, x_{2}, x_{3}, x_{4}\right)$ и $\left(x_{2}, x_{3}, x_{4}, x_{5}\right)$ - для вершин.

ПРЕДЛОЖЕНИЕ 5. Допустим, что $\left\{U_{i}, \varphi_{i}\right\}$ - система открытых множеств и функиий, удовлетворяющих условиям предложения $4 c$ некоторым $\varepsilon>0$. Тогда для любой точки ребра $\{b=0, c=d\}$ (coответственно $\{b=0, d=1\}$, соответственно $\{b=c, d=1\})$ симплекса $\Delta$ и любой области $U_{i}$ әтого покрытия значение соответствующей 


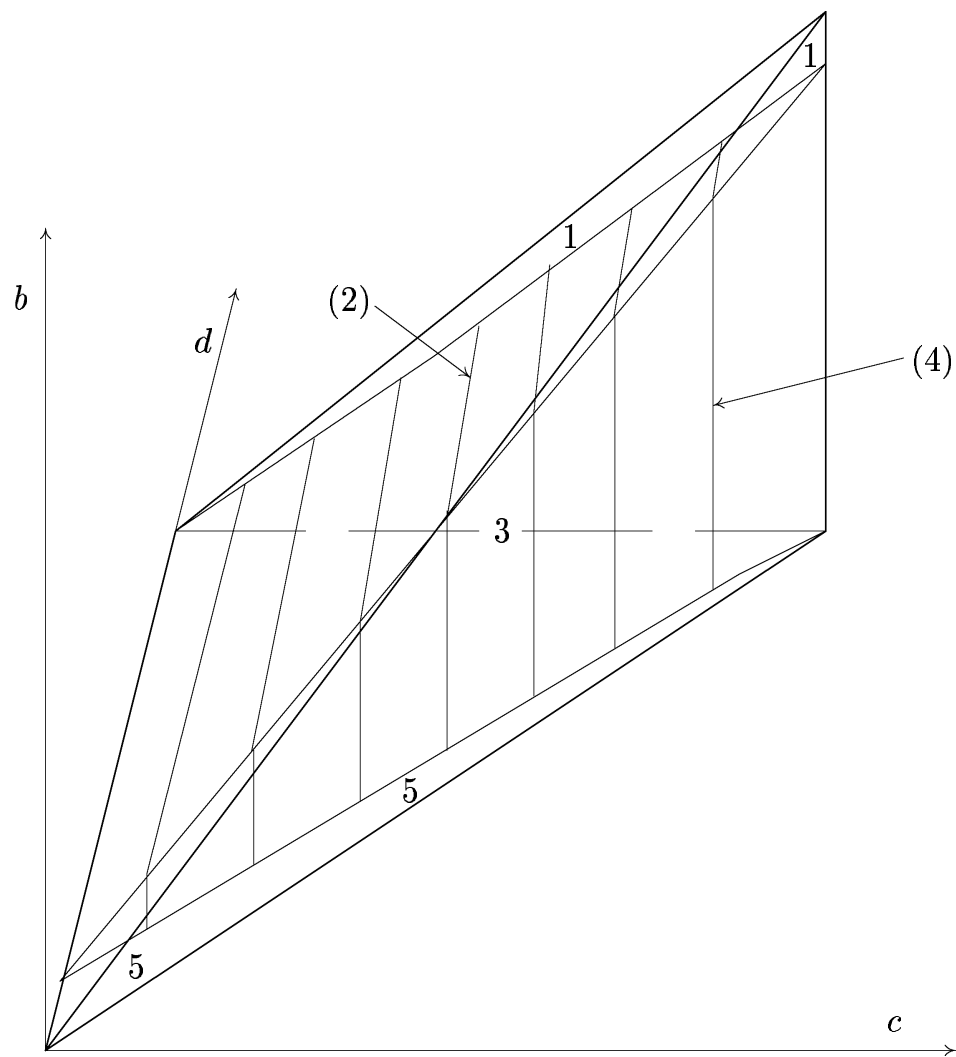

Рис. 3. Полиномы пятой степени с пятью вещественньми корнями

функиии $\varphi_{i}$ в этой точке принадлежит в-окрестности корня $x_{5}$ (соответственно $x_{3}$, соответственно $x_{1}$ ).

Действительно, любые окрестности этих точек в $\Theta$ пересекаются с множеством $V_{1}$ (см. доказательство теоремы 3 ), и (единственные) корни полиномов из этого множества стремятся к значению корня $x_{5}$ (соответственно $x_{3}$, соответственно $\left.x_{1}\right)$, когда эти полиномы стремятся в $V_{1}$ к точкам этих peбер.

Аналогично, если точка $f$ грани $\Delta$ покрыта областью $U_{i}$ нашего покрытия, то соответствуюшее число $\varphi_{i}(f)$ лежит в $\varepsilon$-окрестности объединения некоторых трех корней $f$, номера которых зависят только от грани: например, для грани $\{b=0\}$ эти три корня суть $x_{3}, x_{4}$ и $x_{5}$. А именно, это три корня, которые не исчезают при переходе через эту грань.

3.3. Покрытие $U_{i}$ симплекса $\Delta$. Мы рассечем симплекс $\Delta$ на три куска $\{1\},\{3\}$ и $\{5\}$ поверхностями (2) и (4), как указано на рис. 3 ; опишем 


\section{эти поверхности.}

Сначала проведем два отрезка, соединяюших точку $(1 / 2,1 / 2,1 / 2)$ с точками $(0,0, \delta)$ и $(1-\delta, 1,1)$; здесь $\delta$ настолько мало, что оба эти отрезка лежат в области, где корни $x_{2}$ и $x_{4}$ не более чем $\varepsilon / 4$-разделены. Объединение $T_{3}$ этих отрезков гомеоморфно отрезку [0,1]: этот гомеоморфизм реализуется координатой $c$.

Затем проведем два отрезка $T_{4}$ и $T_{2}$ в гранях $\{b=0\}$ и $\{d=1\}$ соответственно, которые соединяют точки $(0,1,1)$ и $(0,0,1)$ с концами этого отрезка $T_{3}$; функция $c$ является координатой также и на обоих этих отрезках. Для любого значения $\gamma \in[0,1]$ проведем два отрезка в плоскости $\{c=\gamma\}$, соединяюшие соответствующую точку отрезка $T_{3}$ с точками $T_{4}$ и $T_{2}$. Поверхности, заметенные такими отрезками, обозначим через (4) и (2) соответственно.

Далее, покроем $\Delta$ двумя областями $U_{1}(5), U_{2}(5) . U_{1}(5)$ включает всё дополнение к поверхностям (4) и (2) плюс маленькие окрестности точек $(0,1,1)$ и $(0,0,1)$, в которых корни $x_{5}$ и $x_{3}$ (соответственно $x_{3}$ и $\left.x_{1}\right)$ не более чем $\varepsilon / 3$-разделены. Пусть $U_{2}^{\prime}$ - очень узкая окрестность объединения поверхностей (4) и (2), так что любой путь, соединяющий вершины $(0,1,1)$ и $(0,0,1)$ внутри этой окрестности, пересекает окрестность ребра $\{b=c=d\}$, в которой $x_{2}$ и $x_{4}$ не более чем $\varepsilon / 3$-разделены. Выбросим из $U_{2}^{\prime}$ очень маленькие окрестности этих двух вершин, так что остаток не пересекает ребер $\{b=0, c=d\},\{b=0, d=1\}$ и $\{b=c, d=1\}$, но, тем не менее, покрывает множество $\Delta \backslash \overline{U_{1}(5)}$. Этот остаток обозначим через $U_{2}(5)$.

Теперь мы можем определить непрерьвную функцию $\varphi_{1}(5): U_{1}(5) \rightarrow \mathbb{R}$ таким образом, что всюду в области множества $\Delta \backslash((4) \cup(2))$, содержащей вершину $(0,0,0)$ (соответственно $(1,1,1)$, соответственно точку $(0,1 / 2,1))$, она не более чем $\varepsilon / 3$-удалена от значения корня $x_{5}$ (соответственно $x_{1}$, соответственно $x_{3}$ ) соответствуюших полиномов. Действительно, эта функция получается с помошью разбиения единишы из пяти функций, три из которых определены в этих областях и равны значениям этих корней, а оставшиеся две определены в малой окрестности точки $(0,1,1)$ (соответственно $(0,0,1))$ и равны среднему арифметическому корней $x_{5}$ и $x_{3}$ (соответственно $x_{3}$ и $x_{1}$ ).

Аналогично мы определяем непрерьвную функцию $\varphi_{2}(5): U_{2}(5) \rightarrow \mathbb{R}$, которая всюду $\leqslant \varepsilon / 3$-отделена либо от $x_{4}$ (вблизи поверхности $\left.(4)\right)$, либо от $x_{2}$ (вблизи (2)). С помошњю групшы (4) продолжаем наше покрытие множества $\Delta \equiv V_{5} \cap \Theta$ областями $U_{1}(5), U_{2}(5)$ до открытого покрытия $V_{5} \cap\left(\mathbb{R}^{4} \backslash 0\right)$. Наша следующая цель - продолжить это покрытие и функции $\varphi_{1}(5), \varphi_{2}(5)$ на все $\Theta$.

Множество $V_{3} \cap \mathbb{R}^{4}$ всех полиномов вида $(3), d=5$, ровно с тремя корнями является расслоением в смысле Серра, база которого - это простран- 
ство $\Omega_{2}$ всех полиномов степени 4 ровно с двумя вещественньпи корнями $x_{1}, x_{3}$, а слой над таким полиномом $g$ состоит из всех полиномов вида $\left(x-x_{2}\right) g$, где $x_{2} \in\left[x_{1}, x_{3}\right]$. (Над вырожденными $g$, корни $x_{1}, x_{3}$ которых совпадают, эти слои стягиваются в точки.)

Обозначим через $\delta \Omega_{2}$ множество троек $\left\{X, x_{1}, x_{3}\right\} \in \mathbb{R}^{3}$ таких, что $x_{1} \leqslant x_{3}$. Любой гладкий путь, стремящийся внутри $\Omega_{2}$ к полиному с четырьмя вещественными корнями, определяет точку множества $\delta \Omega_{2}: X-$ это новорожденньй двукратньй корень предельного полинома, а $x_{1}, x_{3}-$ два других корня, полученных по непрерывности из вещественных корней полиномов этого пути. (Отметим, что полином вида $(x-\alpha)^{2}(x-\beta)^{2} \in \overline{\Omega_{2}}$, $\alpha \neq \beta$, соответствует двум разньм точкам $\delta \Omega_{2}$.) Наше расслоение над $\Omega_{2}$ продолжается до расслоения над $\Omega_{2} \cup \delta \Omega_{2}$ : слой над тройкой $\left(X, x_{1}, x_{3}\right)$ естественно отождествляется с отрезком $\left[x_{1}, x_{3}\right]$ или, эквивалентно, с множеством полиномов вида $(x-X)^{2}\left(x-x_{1}\right)\left(x-x_{2}\right)\left(x-x_{3}\right), x_{2} \in\left[x_{1}, x_{3}\right]$.

ЛЕмма 4. Существует непрерывное сечение нашего расслоения $\left\{x_{2}\right\}$ над $\Omega_{2}$, удовлетворяющее следующим граничным условиям над мноэиеством $\delta \Omega_{2}$ :

1) если для некоторой точки $g=\left(X, x_{1}, x_{3}\right) \in \delta \Omega_{2}$ число $X$ лежсит межуду $x_{1}$ и $x_{3}$, то очевидное отображсение факторизачии $\mathbb{R}^{4} \backslash 0 \rightarrow \Theta$ переводит слой $\left\{x_{2} \in\left[x_{1}, x_{3}\right]\right\}$ нашего расслоения над $g$ в ломаную линию в $\partial \Delta$, высекаемую из обвединения граней $(b=c) u(c=d)$ некоторой плоскостью вида $\{c=$ const $\}$ ( а именно, const $=\left(X-x_{1}\right) /\left(x_{3}-x_{1}\right)$ ), cм. рис. 3. В частности, его образ пересекает кривую $T_{3}$ в единственной точке. В этом случае значение нашего сечения над $g$ долюно совпадать с прообразом этой точки из $T_{3}$ при этом отображсении факторизачии;

2) если точка $X$ меньше чем $x_{1}$ (соответственно больше чем $\left.x_{3}\right)$, то это же отобрахсение переводит наш слой в отрезок, вьсекаемый на грани $(b=0)$ (соответственно $(d=1))$ плоскостью вида $\{c=$ const $\}$ где const $=\left(x_{1}-X\right) /\left(x_{3}-X\right)$ (соответственно $\left.\left(x_{3}-x_{1}\right) /\left(X-x_{1}\right)\right)$. В этом случае значение нашего сечения над $g$ долэсно совпадать с прообразом точки пересечения әтого отрезка $c$ отрезком $T_{4}$ (соответственно $T_{2}$ ).

Действительно, нет никаких препятствий к построению такого сечения. Определим множество $U_{1}^{\prime}(3) \subset V_{3} \cap\left(\mathbb{R}^{4} \backslash 0\right)$ как дополнение к образу этого сечения и определим множество $U_{1}(3) \subset V_{3} \cap \Theta$ как объединение множества $U_{1}^{\prime}(3) \cap \Theta$ и окрестности ребра возврата, состояшего из всех полиномов $f \in \overline{V_{3}} \cap \Theta$, имеюших корни кратности 3 , настолько малой, что в ней все эти три корня не более чем $\varepsilon / 3$-разделены. Тогда мы можем определить непрерывную функцию $\varphi_{1}(3): U_{1}(3) \rightarrow \mathbb{R}$, значение которой в любой точке $f \in U_{1}(3)$ не более чем $\varepsilon / 3$-отделено от наибольшего $\left(x_{3}\right)$ или 
наименьшего $\left(x_{1}\right)$ корня $f$, а именно, в точках нашего расслоения, которые лежат "ниже" сечения (т.е. в точках слоя $\left\{x_{2}\right\}$ с $x_{2}$ близкими к $x_{1}$ ), эта функция близка к $x_{3}$, а "вьшше" сечения она близка к $x_{1}$.

Область $U_{2}(3) \subset V_{3} \cap \Theta$ определяется как узкая окрестность образа нашего сечения, из которого выброшена очень узкая окрестность ребра возврата, так что $U_{2}(3) \cap \overline{V_{1}}=\varnothing$. Определим $\varphi_{2}(3): U_{2}(3) \rightarrow \mathbb{R}$ как функцию, сопоставляющую любому полиному его средний корень $x_{2}$.

Наконец, положим $U_{1}(1) \equiv V_{1} \cap \Theta$ и определим $\varphi_{1}(1): U_{1}(1) \rightarrow \mathbb{R}$ как функцию, значение которой в каждой точке $f$ равно единственному корню полинома $f$.

Мы построили покрытие топологической сферы $\Theta$ пятью множествами $U_{1}(5), U_{2}(5), U_{1}(3), U_{2}(3)$ и $U_{1}(1)$ и вещественную непрерывную функцию $\varphi_{i}(j)$ на каждом из этих множеств $U_{i}(j)$. По построению любая точка $f$ открытого множества $U_{1} \equiv U_{1}(5) \cup U_{1}(3) \cup U_{1}(1)$ обладает окрестностью $\Lambda$ такой, что все значения всех трех функций $\varphi_{1}(5), \varphi_{1}(3)$ и $\varphi_{1}(1)$ в $U_{1}(5) \cap \Lambda$, $U_{1}(3) \cap \Lambda$ и $U_{1}(1) \cap \Lambda$ соответственно не более чем $2 \varepsilon / 3$-удалены друг от друга. Поэтому с помощью разбиения единицы мы можем построить единую непрерьвную функцию $\varphi_{1}$, определенную на множестве $U_{1}$, значение которой в каждой точке $f$ не более чем $2 \varepsilon / 3$-удалено от значения соответствующей функции $\varphi_{1}(\cdot)$ в этой точке, а следовательно, не более чем $\varepsilon$-удалено от некоторого корня $f$ : конкретнее, это всегда будет нечетньй корень $x_{1}, x_{3}$ или $x_{5}$. Аналогичным образом мы склеиваем вместе функции $\varphi_{2}(5)$ и $\varphi_{2}(3)$ и получаем единую функцию $\varphi_{2}$ на открытом множестве $U_{2} \equiv U_{2}(5) \cup U_{2}(3)$, которая всюду не более чем $\varepsilon$-удалена от некоторого (четного) корня.

Поскольку $U_{1} \cup U_{2}=\Theta$, теорема 4 полностью доказана.

Математический институт им. В. А. Стеклова РАН

Поступило

Независимый московский университет

13.03 .96

\section{СПИСОК ЦИТИРОВАННОЙ ЛИТЕРАТУРЫ}

[1] Smale S. On the topology of algorithms. I // J. Complex. 1987. V. 3. P. 81-89.

[2] Васильев В.А. Когомологии групп кос и сложность алгоритмов // Функцион. анализ и его прилож. 1988. Т. 22. № 3.

[3] Васильев В. А. Топологическая сложность алгоритмов приближенного решения систем полиномиальыых уравнений // Алгебра и анализ. 1989. Т. 1. № 6. C. 98-113.

[4] Vassiliev V.A. Complements of Discriminants of Smooth Maps: Topology and Applications. Revised edition. Translations of Math. Monographs. V. 98. Providence: AMS, 1994. 